\title{
Routing in Wireless Multimedia Home Networks
}

\author{
Hans Scholten, Pierre Jansen and Laurens Hop \\ University of Twente, Department of Computer Science (EEMCS) \\ P.O.B. 217, 7500 AE Enschede, the Netherlands \\ Phone: +31 53489 3733, Fax: +31 534894590 \\ Email: \{scholten, jansen, hop\}@cs.utwente.nl
}

\begin{abstract}
This paper describes an adapted version of the destination sequenced distance vector routing protocol (DSDV) which is suitable to calculate routes in a wireless -real-time- home network. The home network is based on a IEEE $802.11 \mathrm{~b}$ ad hoc network and uses a scheduled token to enforce real-time behaviour to support multimedia streams. The multimedia network protocol works for both single-hop and multi-hop networks, however in the latter case special measures have to be taken to forward streams from node to node and to find routes. Existing routing protocols exhibit non-deterministic behaviour which may interfere with the correct streaming of multimedia. The proposed routing protocol does not rely on flooding, instead it piggy-backs the real-time token and behaves in a predictable manner. Simulation of the routing protocol shows that routes in the network are found in finite time.
\end{abstract}

\section{Index Terms}

Home networks, routing, multimedia streaming, real-time

\section{AD HOC NETWORKS AND ROUTING}

Traditionally, ad hoc networks are considered useful in situations where the necessary infrastructure for centralized administration may not be economically practical or physically possible. Well-known examples of these situations are communication networks established at battlefields or disaster areas. The absence of the need for a base station contributes positively to the ease of installation of an ad hoc network. The size of a managed network is limited by the signal range of the base station. If the network needs to be expanded, multiple base stations are necessary. Ad hoc networks however can easily grow any size, as long as the nodes are placed at distances regular enough to keep the network connected. These properties make ad hoc networks very attractive for deployment at home. However, first the problem of finding routes in finite time must be solved. Routing protocols vary with respect to:

- The way routes are stored: at the originator (source routing) or distributed over the intermediate nodes. The originator must have some information about the destination, like the number of hops needed for transmission and the first node to send the data to.

- The moment the routing protocol takes the initiative for finding a route to a destination. Reactive routing protocols attempt to discover routes only on demand by flooding a query in the network. Proactive routing protocols are based on periodic exchange of control messages. Nodes calculate routes when new control data arrives. Routes are available immediately when needed.

The following sections introduce some routing protocols designed for ad-hoc networks.

\section{A. Dynamic Source Routing}

In this protocol [1], the complete route is determined by the source node (originator). If necessary, the originator performs a routing request. This request is flooded over the network, eventually reaching the destination. While the request is forwarded, intermediate nodes attach their address to it, resulting in a

This work is sponsored by the Netherlands Organization for Scientific Research (NWO) under grant number 612.060.111, and by the IBM Equinox programme. 
complete route which can be extracted from the request by the destination. The destination sends a route reply, which contains the route discovered, to the originator (using the reverse route, or by performing another routing request if routes are not assumed to be bi-directional). Discovered routes are cached for future use.

\section{B. Ad-hoc On-demand Distance Vector routing}

The routing table of this protocol [2] only lists the number of hops, the address of the first intermediate node towards the destination, and some information for administrative purposes. Routes are stored distributedly: the route a message follows is established one hop at a time. Each node is responsible for forwarding the message into the right direction. As with source routing, a routing request is flooded over the network. The route travelled so far is not stored in the request, but in the routing tables of the intermediate nodes as reverse routes towards the originator. This route will be used when sending the route reply from the destination back to the originator. While forwarding this route reply to the originator, the route towards the intended destination is stored in the intermediate routing tables. As soon as the route reply message reaches the originator, a full bi-directional route is established.

\section{Destination Sequenced Distance Vector Routing}

DSDV [3] is the predecessor of AODV (see previous section) and is a proactive routing protocol designed for ad-hoc networks. Instead of performing routing requests on demand, nodes periodically advertise (broadcast) their routing tables to their direct neighbours. To reduce overhead, the information contained in this advertisement may be limited to incremental updates. Nodes also perform routing table advertisement when the table significantly changes (e.g., when an advertisement from a neighbour leads to new or updated routes). Sequence numbers are kept to monitor the freshness of the routes.

\section{Link State Routing}

The concept of link state routing is based on periodically broadcasting a nodes neighbour list to all other nodes in the network. Since every node will obtain complete knowledge of the network topology, algorithms like Dijkstra's shortest path algorithm can be used to compute optimal routes. It is a proactive routing protocol. The Optimised Link State Routing protocol (OLSR) [4] optimises this process by compacting the size of control information, and furthermore, reducing the number of retransmissions to flood this information in the entire network by means of a technique called multipoint relaying. Control messages broadcasted by a node are only forwarded by multipoint relays (MPRs) of that node: a subset of neighbour nodes which is chosen such that all nodes in the two hop neighbourhood are covered. As soon as enough information is available, the minimal subset can be computed.

\section{E. Zone Routing Protocol}

The Zone Routing Protocol [5] belongs to the group of hybrid protocols, combining the best of proactive and reactive behaviour. A node learns the topology of its own routing zone, which includes all nodes whose distance (in hops) is at most a predefined zone radius, by means of a truncated version of a proactive routing protocol, like DSDV. For obtaining routes to destinations beyond its own zone, a node broadcasts a query to its peripheral nodes, which is similar to the reactive routing approach. By omitting all nodes within the routing zones, the number of control messages are reduced substantially.

\section{F. Temporally-Ordered Routing Algorithm}

TORA [6] is a distributed routing protocol based on a link reversal algorithm. It is designed to discover routes on demand, provide multiple routes to a destination, establish routes quickly, and minimize communication overhead by localizing algorithmic reaction to topological changes when possible. Route optimality (shortest-path routing) is considered of secondary importance, and longer routes are often used to avoid the overhead of discovering newer routes. 


\section{G. Broadcasting}

Broadcasting (i.e. sending a message to all nodes in a network) is a common operation in network applications. In ad-hoc networks, such an operation is expected to be executed more frequently, particularly when finding a route to another node. The most straightforward way of broadcasting is by flooding, where a message is rebroadcast by every node that receives it. The majority of the routing protocols described in the previous sections indeed utilize flooding to distribute topology information and/or routing requests. In the paper by Tseng et al. [7] the broadcast storm caused by flooding is analysed carefully, by simulation and theoretical analysis. Drawbacks of flooding include:

- Redundant rebroadcasts: When a node decides to rebroadcast a broadcast message to its neighbours, it is likely that all neighbours already have the message. Depending on the geographical placement and the average number of neighbours, the expected additional coverage (the probability a node is reached that has not yet received the message) is up to 61 percent. If the message is already rebroadcast by a few neighbours, the expected additional coverage even drops below 5 percent.

- Contention: After a node broadcasts a message, if many of its neighbours decide to rebroadcast the message, these transmissions may severely contend with each other. The neighbours receive the broadcast simultaneously and are likely to reside in each others neighbourhood. The probability of contention of two nodes is 59 percent, for six nodes this increases quickly over 80 percent.

- Collision: Because of the deficiency of the back off mechanism, the lack of an RTS/CTS dialogue and the absence of collision detection, collisions are more likely to occur and cause more damage.

Real-time networks require transmission time to be predictable. Flooding however, is very unpredictable with respect to timing behaviour. The time needed for flooding depends on the network diameter. The diameter is an upper bound for the number of times a message is expected to be rebroadcast. The diameter of a network is initially an unknown parameter. Extracting topology information from the network in order to calculate this measure, flooding is required. The total number of nodes in the network could be used as an upper bound for the number of rebroadcasts. However, this metric might also not be available. The broadcast storm problem described above introduces severe non-deterministic behaviour. [8] proposes a protocol in which time is divided into rounds (slots). In each round, a node acts either as a transmitter or as a receiver. A node receives a message in a specific round if, and only if, in that round it acts as a receiver and exactly one of its neighbours acts as a transmitter. Which node will act as a transmitter in a specific round is pre-calculated at initialisation and is mobility-independent (topology-independent). The protocol requires a synchronized clock and knowledge of $\mathrm{n}$, the number of nodes in the network, and $\Delta$, the maximum degree of a node (maximum number of neighbours), which is assumed to be less than $\mathrm{n}$ 1. The protocol does not need knowledge of a node's neighbourhood. It guarantees collision-free flooding in a predictable time. In home environments, nodes are expected to be located close to each other and it is plausible that in many cases $\Delta=\mathrm{n}-1$. As a result, it will take $\mathrm{n}$ rounds before a message is transmitted one hop further away from the source. For an operation that is to be executed as frequently as the routing process, the delay introduced by this algorithm is unacceptable.

\section{H. Real-time ad-hoc networks}

Routing in a real-time network is often referred to as quality of service-routing (QoS routing). Examples of such networks are given by Lin and Gerla [9] and by $\mathrm{Wu}$ and Chuang [10]. The systems proposed there are not token based and perform bandwidth reservation around single nodes. The challenge they are facing is finding routes through nodes which have enough available bandwidth. They combine the use of routing protocols, bandwidth reservation protocols, and collision avoidance protocols. Although these mechanisms do guarantee the requested bandwidth, hard deadlines are not always explicitly taken into account. A token based protocol like RTnet (see [11]) is inherently collision-free and much of the collision avoidance should not be necessary. However, one major disadvantage of the token approach is that there is always only one token, even if the network grows so large that several groups of nodes 
could communicate independently without interfering with each other. Protocols which utilize bandwidth reservation around each distinct node, take advantage of this situation.

\section{REAL-TIME MULTI-HOP ROUTING}

In this section we will introduce a routing protocol which can be used in a real-time scheduled token home network. The proposed routing protocol is a supplement to the existing RTnet protocol [11] and is an adaptation of the DSDV routing protocol as described in the previous section. RTnet is based on a token that is scheduled by an earliest deadline first (EDF) scheduler. In principle EDF can utilize 100 percent of the network bandwidth minus overhead. Whenever a node has the token it may send one data packet, e.g. a set of video or audio frames. Then the token is send to the node next in the schedule. Up to 80 percent of the bandwidth is reserved for real-time data transfer. The remaining bandwidth is used to send the token around in a round-robin manner for network maintenance, such as discovering new nodes and token management.

\section{A. Assumptions}

Requirements on genuine ad hoc networks are virtually impossible to hold in real-time networks. For example, mobility of nodes introduces a non-deterministic network topology, making it impossible to guarantee routes to be valid for a long enough time. However, in home network environments, assumptions can be made on node availability, mobility, and distance. These assumptions loosen requirements a lot, allowing new strategies to be developed.

- Nodes are mobile, however, their movement is limited. Most devices have a more or less fixed position in the house, e.g. TVs do not change position often.

- Distance between nodes is limited. The signal range of a node is not much less than the size of the house. As a result, the diameter of the network will be small, in most cases even one.

- Some rerouting time after the occurrence of a broken link is allowed.

- The number of nodes in the network is finite and not very large, a few dozens at most.

- The token visits each node frequently, while nodes do not hold the token for a very long time.

\section{B. Real-time DSDV}

The multi-hop RTnet extension combines the DSDV routing protocol with the use of a token. The idle time, in which the token circulates in the network, is used to exchange routing information. All nodes will learn every route to each other. The mechanism has the following properties:

- It does not perform any flooding. Routing information is exchanged using single broadcasts and unicasts between neighbouring nodes. Flooding is a major problem in a real-time network in which unpredictable collisions must be avoided at all cost.

- Only the node possessing the token transmits data and consequently the protocol is completely collision free.

- Routes are either available immediately when requested, or not available until a later stage. In any case the timing behaviour of the protocol is completely predictable.

- In a network of a finite number of nodes, every route between arbitrary nodes is constructed in finite time. After a topological change, the network stabilizes itself in finite time. Under the assumptions discussed above this time will be short enough for practical use at home.

The original protocol prescribes advertisements whenever a node discovers a significant topology change, for instance when it receives an advertisement from its neighbour. Therefore, a topology change will cause a cascade of advertisements through the network. This is similar to flooding, which is a chain reaction of broadcasts. The assumptions above alleviate the need for an immediate advertisement after receiving an advertisement from a neighbour. If a node postpones this advertisement until it has the token, the routing time will be substantially larger, but this is acceptable. But, because only the node with token 


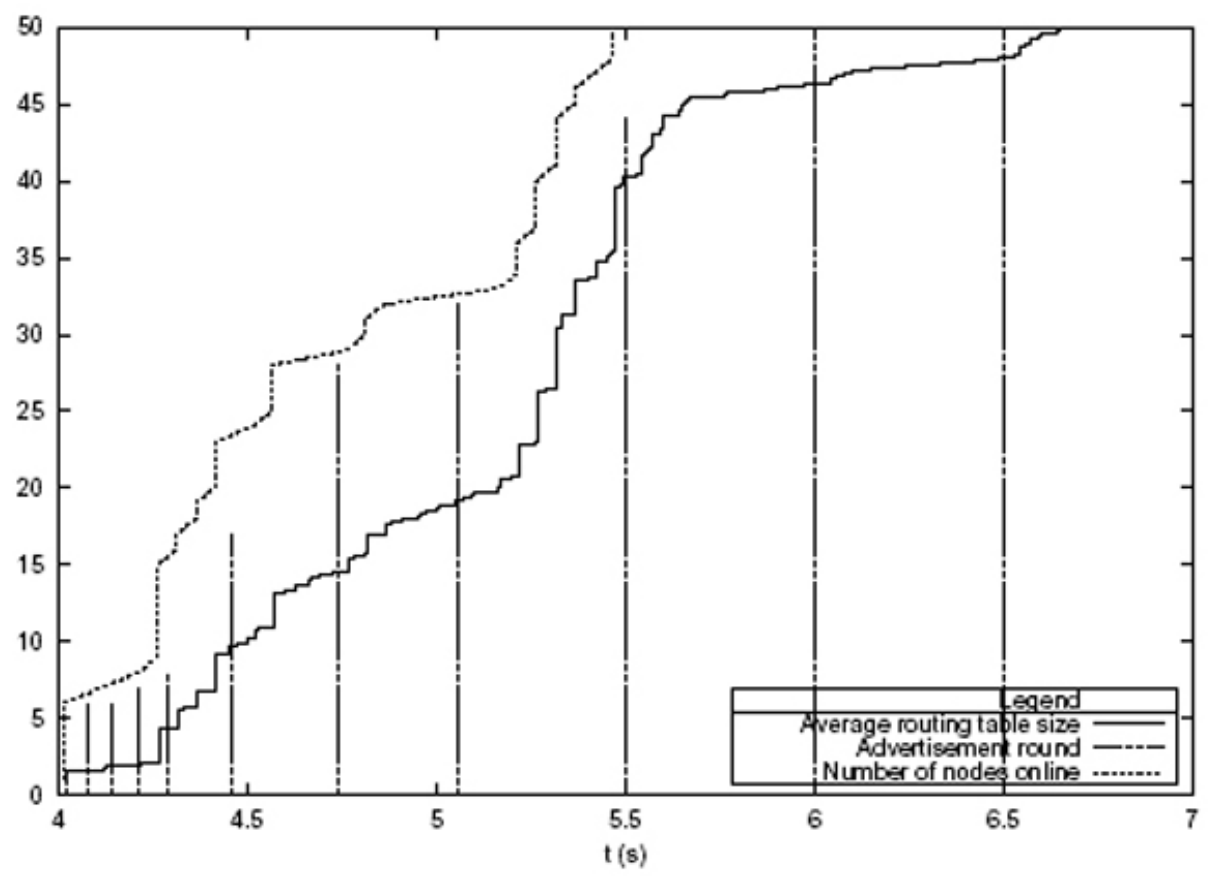

Fig. 1. Route discovery at range 100

may send, no flooding or collision occurs. Simulations show that routing time is indeed suitable for home environments. The route discovery process takes place when all real-time data streams are idle, which is the case during the 20 percent slack time available for non real-time traffic. During this phase, the token travels the network in a round-robin manner, i.e. every node receives the token by turns. The address of the next node to send the token to is extracted from a list of known addresses which is contained in the token. When all nodes from the list have received the token, a round is completed and a new round is started. At a certain moment during the time a node holds the token, it advertises its routing table by broadcasting it to its direct neighbours. The upper bound for the time needed for this advertisement is known in advance and can be taken into account for calculating the remaining time left for the transmission of other non real-time data. It can be proven that a route of $\mathrm{n}$ hops is learnt after at most $\mathrm{n}$ rounds. The upper bound is equal to the number of nodes in the network. The average number of rounds to find an $\mathrm{n}$ hop route is equal to $\Delta$. Nodes receiving the advertisement update their routing table. Every entry from the advertised routing table is compared against an entry from the local routing table to the same destination (if available). If the destination is not yet in the local routing table, an entry will be added. If the entry from the advertised routing table is better than the local entry, the local entry will be updated.

\section{SimULATION}

The multi-hop RTnet protocol is simulated in OMNeT++, a discrete event simulation system developed by Varga [12]. This simulation includes all phases of the network, including initialisation. The network needs 4.015 seconds to start up. Then the route discovery process begins. Figure 1 shows the number of nodes that are online, i.e. the nodes to where a route is found. The signal range is 100 , which corresponds with a network with $\Delta=12$. A vertical bar indicates the start of a new routing table advertisement round. The height of these bars shows the number of nodes participating in the previous round. Observe that the distance between rounds increases with the number of online nodes until all routes are found. From that moment on no new routes are added to the routing table, but the existing ones converge to their optimal -lowest- values. This is shown in figure 2, where the longest route takes 12 hops, which is equal to the diameter. The network is stable as soon as all routes become steady. This happens in finite time and is bounded by the network diameter. Table I summarises the results of the simulation. 


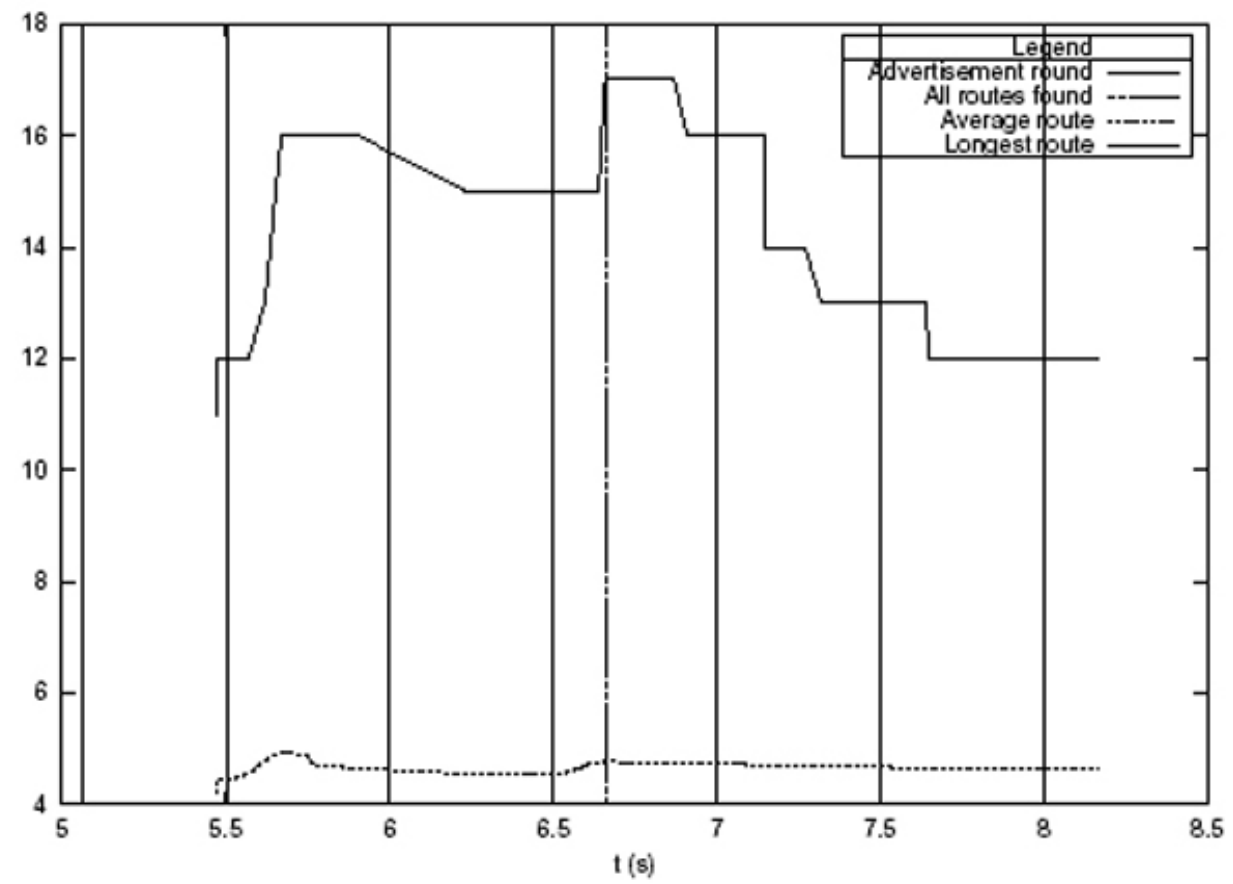

Fig. 2. Route convergence at range 100

\begin{tabular}{|lr|rrr|}
\hline signal range & $\Delta$ & $\begin{array}{r}\text { all nodes online } \\
(\mathrm{sec})\end{array}$ & $\begin{array}{r}\text { all routes found } \\
(\mathrm{sec})\end{array}$ & $\begin{array}{r}\text { stable } \\
(\mathrm{sec})\end{array}$ \\
\hline 100 & 12 & 1.452 & 2.645 & 4.155 \\
125 & 8 & 1.700 & 2.255 & 2.575 \\
150 & 6 & 0.750 & 1.075 & 1.795 \\
200 & 4 & 0.450 & 0.545 & 0.955 \\
300 & 3 & 0.100 & 0.135 & 0.695 \\
400 & 2 & 0.100 & 0.105 & 0.586 \\
unlimited & 1 & 0.00009 & 0.005 & 0.486 \\
\hline
\end{tabular}

TABLE I

SIMULATION RESULTS AT VARIOUS SIGNAL RANGES

\section{CONCLUSION}

The adapted routing protocol performs acceptable at larger signal ranges, thus reducing the diameter of the network. With a signal range of 100 and diameter 12, which may be considered very large for a home network, performance is poor. However, even in this complex topology all routes are discovered within 3 seconds and are stable in approximately 4.1 seconds. This is very acceptable with regards to the formulated requirements. The most important result however is the predictability of the protocol, which makes it suitable for real-timestreaming multimedia networks.

\section{REFERENCES}

[1] D.B. Johnson and D.A. Maltz. Dynamic Source Routing in Ad-hoc Wireless Networks, Mobile Computing, T. Imielinksi and H. Korth, Eds., pp.153-181, Kluwer Academic Publishers, 1996.

[2] C.E. Perkins and E.M. Royer. Ad-hoc On-Demand Distance Vector Routing, Proceedings of the 2nd IEEE Workshop on Mobile Computing Systems and Applications, pp. 90-100. Feb. 1999.

[3] C.E. Perkins and P. Bhagwat. Highly Dynamic Destination-Sequenced Distance-Vector Routing (DSDV) for Mobile Computers. ACM SIGCOMM Computer Communication Review, vol 24, no. 4, pp. 234-244, Oct. 1994.

[4] P. Jacuet, P. Muhlethaler, T. Clausen, A. Laouiti, A. Qayyum, L. Viennot. Optimized Link State Routing Protocol for Ad Hoc Networks, IEEE INMIC Pakistan, pp. 62-68, Dec. 2001. 
[5] Z.J. Haas. A New Routing Protocol for the Reconfigurable Wireless Networks. IEEE International Conference on Universal Personal Communication (ICUPC), vol. 2, pp. 562-566, Oct. 1997.

[6] V. Park and S. Corson. A Highly Adaptive Distributed Routing Algorithm for Mobile Wireless Networks. Proceedings of IEEE Conference on Computer Communications Infocom 97, vol. 3, pp. 1405-1413, Apr. 1997.

[7] Yu-Chee Tseng and Sze-Yao Ni and Yuh-Shyan Chen and Jang-Ping Sheu. The broadcast storm problem in a mobile ad hoc network, Wireless Networks, vol. 8 no. 2/3, pp.153-167, Mar. 2002.

[8] S. Basagni and D. Bruschi and I. Chlamtac. A Mobility-Transparent Deterministic Broadcast Mechanism for Ad Hoc Networks. IEEE/ACM transactions on networking, vol. 7, no. 6, Dec. 1999.

[9] C.R. Lin and M. Gerla. Real-time support in multihop wireless networks. Wireless Networks, vol.5, no.2, pp.125-135, Mar. 1999.

[10] H.K. Wu and P.H. Chuang. Dynamic QoS Allocation for Multimedia Ad Hoc Wireless Networks, Mobile Networks and Applications, vol.6, no. 4, pp. 377-384, Aug. 2001.

[11] J. Scholten, P. G. Jansen, F. T. Y. Hanssen, P. H. Hartel, T. Hattink, and V. Sundramoorthy, Multimedia QoS in low-cost home networks, in 27th Annual IEEE Conference on Local Computer Networks (LCN), Tampa, Florida: IEEE Computer Society Press, Los Alamitos, California, pp. 327-328, Nov 2002.

[12] A. Varga. OMNeT++. http://www.omnetpp.org. 